\title{
Efficient Multi-Modal Fusion with Diversity Analysis
}

\author{
Shuhui Qu, Yan Kang, Janghwan Lee \\ \{shuhui.qu;yan.kang;jake.ee\}@samsung.com \\ Samsung Display American Lab \\ San Jose, California, U.S.A.
}

\begin{abstract}
Multi-modal machine learning has been a prominent multi-disciplinary research area since its success in complex real-world problems. Empirically, multi-branch fusion models tend to generate better results when there is a high diversity among each branch of the model. However, such experience alone does not guarantee the fusion model's best performance nor have sufficient theoretical support. We present the theoretical estimation of the fusion models' performance by measuring each branch model's performance and the distance between branches based on the analysis of several most popular fusion methods. The theorem is validated empirically by numerical experiments. We further present a branch model selection framework to identify the candidate branches for fusion models to achieve the optimal multi-modal performance by using the theorem. The framework's effectiveness is demonstrated on various datasets by showing how effectively selecting the combination of branch models to attain superior performance.
\end{abstract}

\section{CCS CONCEPTS}

- Computing methodologies $\rightarrow$ Artificial intelligence; Ensemble methods.

\section{KEYWORDS}

multi-modal, neural networks, diversity analysis, architecture selection

\section{ACM Reference Format:}

Shuhui Qu, Yan Kang, Janghwan Lee. 2021. Efficient Multi-Modal Fusion with Diversity Analysis. In Proceedings of the 29th ACM Int'l Conference on Multimedia (MM '21), Oct. 20-24, 2021, Virtual Event, China. ACM, New York, NY, USA, 8 pages. https://doi.org/10.1145/3474085.3475188

\section{INTRODUCTION}

Multi-modal machine learning has received much attention from academia and industry since its successful application to complex real-world problems in areas including multi-media, computer vision, speech recognition, and text processing $[17,21]$. The real-world concepts are described by different views or data types; in other words, naturally multi-modal[21]. For example, a medical record for a broken arm can be recorded and diagnosed with a collection of text, X-ray, CAT scan, and MRI images [33]; Social network users

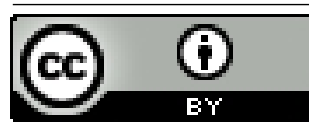

This work is licensed under a Creative Commons Attribution International 4.0 License.

MM '21, October 20-24, 2021, Virtual Event, China.

(C) 2021 Copyright held by the owner/author(s).

ACM ISBN 978-1-4503-8651-7/21/10.

https://doi.org/10.1145/3474085.3475188 post through video, audio, image, and text [8]. Recently, some multimodal tasks have also achieved great success in tackling artificial intelligence challenges, such as visual question answering, image captioning, vision-language navigation through the development of methods that combine different model architectures. [3, 8, 40]

Most of the existing research in multimodal machine learning focuses on mapping representation from one modality to another modality or creating a shared representation between modalities by solving an auxiliary task $[5,18]$. More specifically, researchers carefully design the fusion model to optimize toward a higher objective function: 1) design the model to extract information by learning the representation for each modality; 2) design the fusion methods to map multiple heterogeneous representations to a common feature space[8]. However, one question remains unclear to the community: how to select the branch model for each modality to build the final fusion model? For example, for an audio-visual multimodal classification task, we fuse the visual branch selected from a set of visual models (ResNet, EfficientNet,...) and audio branch selected from the audio models (Gated Recurrent Neural Networks (GRNN), Convolutional Self-attention Network (CSN),...). How to select the branch model for visual and audio modality to build the fusion model that generates the highest accuracy (ResNet+GRNN, ResNet+CSN, EfficientNet+GRNN, or other combination)? Empirically, such a multi-branch fusion model achieves a higher objective function when there is a high diversity among branches. However, there lacks a theoretical and empirical explanation that can systematically explain such experience.

In this paper, we present a multi-modal diversity analysis to address the question mentioned above. We especially focus on the multi-modal multi-class classification problem. We hypothesis that the distance between each branch model indicates the complementary information provided by each other; the more complementary information, the higher the accuracy improvement of the final fusion model can be achieved. Our proof shows that the final fusion model's performance is represented by a function of the distance between branches and the individual branch models' performance on the objective function for the late and middle fusion strategies. The accuracy improvement of the final fusion model has a log-linear correlation with the distance measure. Based on our theoretical analysis, we propose a framework to select a branch model for each modality to fuse toward the higher objective function. This framework first learns the parameters in the fusion model's performance estimation function from our theorem. Using the function, we then can estimate fusion models' performances and rank models based on their estimated performance. The final fusion model to train is selected according to the ranking of such estimated performance. 
We apply our framework to several popular multi-modal tasks: kinetic sound prediction (Kinetic-Sound dataset[19]), e-Commerce advertisement (Ferramenta dataset[14]), sentimental analysis (CMUYoutube, CMU-MOUD, CMU-MOSI dataset[43]). These datasets consist of multiple data types, including video, audio, image, and text. Our experiment demonstrates our hypothesis that multi-branch fusion models' performance gain is log-linear correlated to the distance between branches; The final fusion model's performance also depends on the branch model's performance. Our proposed framework is providing the functionality to efficiently select the combination of branch models and, as the results, the accuracy of the selected final fusion model using our framework is improved compare to the previous fusion models regardless of the fusion techniques across these tasks. In total, we believe our framework provides a first look into the branch model selection for multimodal analysis, providing a practical alternative to predict the performance of the fusion model.

This paper's contributions come from multi-folds:

- We theoretically present the fusion model's performance as a function of the branch model's distance and branch models' performance.

- We empirically show strong log-linear correlation between fusion model's performance improvement and the branch model's distance across various datasets.

- Based on our theorem, we propose a framework that efficiently finds the branch combination to fuse for optimal performance.

- We validate the effectiveness of our proposed framework across several popular datasets by achieving competitive results.

The rest of the paper is organized as follows: prior related work is shown in section 2 . We then describe our theoretical analysis and our model selection framework in section 3. The experimental results are shown in section 4 . We then conclude the paper in section 5 .

\section{LITERATURE REVIEW}

The multi-modal machine learning area research aims to integrate information from the heterogeneity of multiple data sources. Learning from multi-modal data requires to capture corresponding representation for each modality and an in-depth analysis of the integration phenomenon[8]. Traditionally, multi-class multi-modal classification focuses on representation learning to understand each modality, information alignment to align elements from different representations, fusion to join information from various sources to perform the classification task[20].

Representation learning provides a good generic prior to the machine learning tasks[10]. A good representation is critical to the final performance of machine learning models. Various works have been conducted to extract the best feature representations for later stage training. Typically, [10] identify useful representation features by smoothness, sparsity, and the similarity concept from each data modality. [26, 30,31] also specify the desired property for multi-modal representation: each modality provides complementary information with other modalities; the representation should provide the missing modality given the observed ones. Typically, most representation learning methods consider extracting the representation from one single modality at one time[6]. Some works try to deal with the feature alignment or coordination of features to maximize the correlation, structured correlation, similarities such as CCA[32], DCCA[2], and KCCA[34]. Many works also deal with translation between modalities[12, 44]. For example, [13] are using cross-modal retrieval to model the correlation between modality models.

There is also a wide range of research in model distance learning. Use distance learning to divert the representation to improve the performance has also been widely studied[22, 30], e.g., in the generative models, GAN and VAE model $[1,9,11]$. In these works, they try to calculate the mutual information loss between the generated samples and the real samples and hope to increase the generated images' quality [9]; however, it is hard to measure the improvement of the quality of the generated image quantitatively.

Other multi-modal research has explored either shallow [39] or deep[28] metric learning for data with multi-sources, but their motivation is to train a multimodal model to measure the distance between instances for better representing the feature space and facilitating image retrieval or further applications on the embeddings. Our motivation is to measure the distance between the singlemodality models and efficiently select the most complementary pairs to gain the best final performance improvement. Also, in [28], the authors share a similar two-stage training strategy with us, but they use unsupervised learning in the first stage and incorporate supervision on the pairwise sample similarity in the second stage. As we don't use any pairwise similarity labels, we are different from them in both the distance measure subject and the training strategy.

Fusion technique is also one of the most popular topics in multimodal machine learning, with extensive work in early, late, middle fusion approaches[6, 27, 37]. In recent work[8], the line between multimodal representation and fusion has been blurred for models such as deep neural networks where representation learning is interlaced with classification or regression objectives. People design various fusion layers to fuse the model toward higher accuracy[23, 41, 42].

However, most of these models work solely on the determined architecture of branch models, i.e., study the fusion technique on the fixed architecture of the visual branch and the fixed architecture for the audio branch. For example, researchers study the fusion layer that fuses the output of the visual ResNet and the output the audio CSN. However, few of them study how to select different architectures to fuse. This is probably due to the fact that the performance of the fusion model cannot be foreseen until the model is trained. Studying such relation costs a lot of computational resources[24, 42]. Empirically, people select the architecture for branch models with a high diversity among branches. However, there is no theoretical analysis to provide such a guarantee.

Thus, in this work, to answer these questions, we provide the theoretical and empirical support to guide us to understand the connection between branch model combination and the performance and how to select the best model for each branch to fuse for most fusion techniques efficiently. 

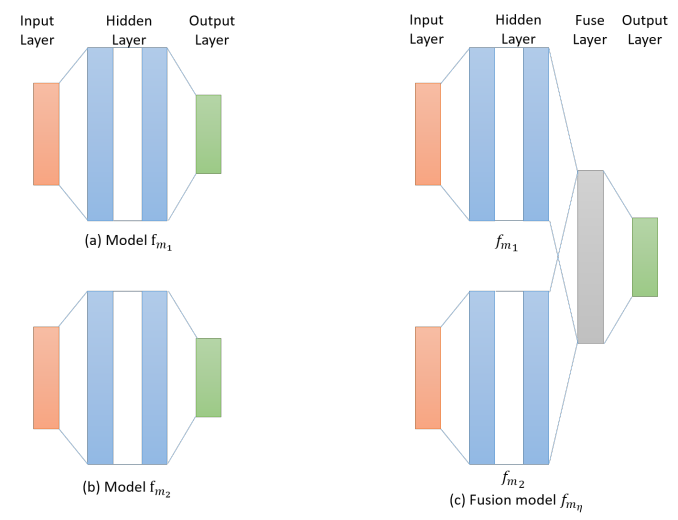

Figure 1: An example of two branch model $f_{m_{1}}, f_{m_{2}}$ and the fusion model $f_{\eta_{m_{1}, m_{2}}}$. In this example, (a) is the branch model $f_{m_{1}}$ that is trained with data modality 1 ; (b) is the branch model $f_{m_{2}}$ that is trained with data modality 2 ; (c) is the fusion model whose branches are initialized by $f_{m_{1}}$ and $f_{m_{2}}$

\section{METHODOLOGY}

This section describes the theoretical analysis of the multi-branch fusion model's performance and branch model selection framework that identifies the branch candidates to fuse. We formally prove the fusion models' performance as a function of branch models' performances and the distance between branches. Based on the theorem, we present the branch model selection framework to select the candidate branch to fuse according to the ranking of the predicted final model's performance. An example of the fusion model with two branches is shown in Figure.1.

\subsection{Problem Formulation}

A dataset, $D=\left\{\left(x_{i, j} j_{i=1, \ldots, M}, y_{j}\right)\right\}_{j=1,2,3, \ldots}$, consists of data with $M$ modalities and label $y$. We obtain a set of pretrained models $\left\{f_{m_{i}, \theta_{i, k}}\left(x_{i}\right)\right\}_{k=1, \ldots, K_{i}}$ for each modality $m_{i}$, where $\theta$ represents training architectures and hyper-parameters. The final fusion model selects one pretrained model $f_{m_{i}, \theta_{i, k}}\left(x_{i}\right)$ for modality $m_{i}$ as its $i^{t h}$ branch, and fuse all $M$ branches using the fusion method $\eta: \hat{y}_{\eta_{m}}=$ $f_{\eta_{m}}\left(\left\{f_{m_{i}, \theta_{i, k}}\left(x_{i}\right)\right\}_{i=1, \ldots, M}\right)$. We need to find the branch model combination $\left\{\theta_{i, k} \mid 1 \leq k \leq K_{i}\right\}_{i=1, \ldots, M}$ such that the highest performance with minimized loss, $L\left(f_{\eta_{m}}\left(\left\{f_{m_{i}, \theta_{i, k}}\left(x_{i}\right)\right\}_{i=1, \ldots, M}\right), y\right)$, of the final fusion model is achieved.

\subsection{Model Distance}

Empirically, the higher the diversity among branches of the fusion models, the fusion model tends to generate better performance. Thus, we hypothesize that the diversity measure between the singlemodality(branch) models explains the amount of complementary information gathered from each branch. The higher the diversity measure, the more complementary information, and thus leads to higher performance improvement by the fusion model. We define the model distance as a representation of the diversity of the models.

Definition 1: (Model distance) Given two pretrained branch models $f_{m_{1}}\left(x_{1}\right)$ (shorthand by $\left.f_{1}\right)$ and $f_{m_{2}}\left(x_{2}\right)\left(f_{2}\right)$ and dataset $D$, the model distance between $f_{1}$ and $f_{2}$ is defined as $d_{m}=d_{m}\left(f_{1}, f_{2}, D\right)$ with selected distance measurement, such as the Joint Entropy (when $M \leq 2$ ).

Note that When $M>2$, the distance is defined as $d_{m}=d_{m}\left(\left\{f_{i}\right\}_{i=1, \ldots, M}, D\right)$, and measured by total correlation.

\subsection{Correlation Between Model Distance $d_{m}$ and Information Gain of The Fusion Model}

We use the mutual information as the proxy to represent the information gain $[7,22]$, which indicates the model performance. The mutual information $I\left(f_{1}, y\right)$ between the output of model $f_{1}$ and true label $y$ is defined as follows:

$$
I\left(f_{1}, y\right)=H\left(f_{1}\right)+H(y)-H\left(f_{1}, y\right)
$$

where $H$ is the entropy or joint entropy depending on how many variables are input.

The mutual information $I\left(f_{2}, y\right)$ between the output of model $f_{2}$ and true label $y$ is,

$$
I\left(f_{2}, y\right)=H\left(f_{2}\right)+H(y)-H\left(f_{2}, y\right)
$$

The mutual information $I\left(f_{1}, f_{2}\right)$ between the output of model $f_{1}$ and the output of model $f_{2}$ is,

$$
I\left(f_{1}, f_{2}\right)=H\left(f_{1}\right)+H\left(f_{2}\right)-H\left(f_{1}, f_{2}\right)
$$

The mutual information between the output of the fusion model $f_{\eta_{m_{1,2}}}\left(x_{1}, x_{2}\right)$ (shorthand as $\left.f_{\eta_{m_{1,2}}}\right)$ and true label y, $I\left(f_{\eta_{m_{1,2}}}, y\right)$ is,

$$
I\left(f_{\eta_{m_{1,2}}}, y\right)=H\left(f_{\eta_{m_{1,2}}}\right)+H(y)-H\left(f_{\eta_{m_{1,2}}}, y\right)
$$

Our proof is based on three major fusion methods, which have been widely applied in many applications[14].

3.3.1 Late fusion method. The late fusion method is also considered as the weighting method. It can be written as:

$$
f_{\eta_{m_{1,2}}}=\beta_{1} f_{1}+\beta_{2} f_{2}
$$

where $\beta_{1}, \beta_{2} \in[0,1]$ and $\beta_{1}+\beta_{2}=1$ are the weighted combination factors for models $f_{m_{1}}$ and $f_{m_{2}}$.

3.3.2 Middle fusion method. Instead of fusing at the output layer, The middle fusion method fuses the branch models at the middle layers. Without the loss of generality, the first branch model $f_{1}$ is constructed as:

$$
f_{1}=\operatorname{Softmax}\left(w_{1}^{T} f_{m_{1}, l}\right)
$$

where $l$ denotes the last latent layer of the deep network model $f_{m_{1}}$, and $w_{1}^{T}$ is the transformation matrix. Similarly, for the second branch

$$
f_{2}=\operatorname{Softmax}\left(w_{2}^{T} f_{m_{2}, l}\right)
$$

The fusion operation is written as:

$$
f_{\eta_{m_{1,2}}}=\operatorname{Softmax}\left(\left(w_{1}^{T} f_{m_{1}, l}+w_{2}^{T} f_{m_{2}, l}\right)\right)
$$

3.3.3 Nonlinear middle fusion method. The nonlinear middle fusion method adds nonlinear operation, e.g., Relu[25], to the middle fusion layer. This fusion method is one of the most popular fusion methods since it maintains the model's nonlinear property after the fusion layer. The first branch model, $f_{1}$ is constructed as follows:

$$
f_{1}=\operatorname{Softmax}\left(w_{1}^{\prime}{ }^{\prime} \operatorname{Relu}\left(w_{1}^{T} f_{m_{1}, l}\right)\right)
$$



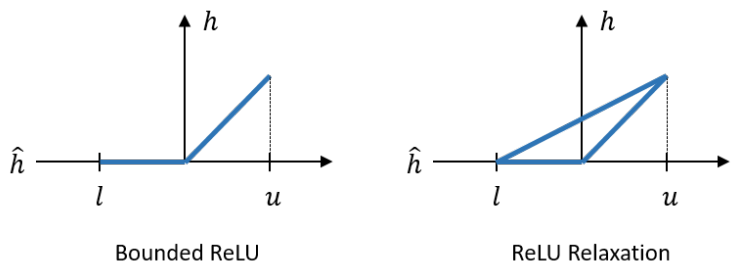

Figure 2: The convex relaxation of the ReLU activation

where $l$ denotes the last latent layer of the deep network model $f_{m_{1}}$, and $w_{1}^{\prime}, T, w_{1}^{T}$ are the transformation matrices. Similarly, the second model is as:

$$
f_{2}=\operatorname{Softmax}\left(w_{2}^{\prime}{ }^{T} \operatorname{Relu}\left(w_{2}^{T} f_{m_{2}, l}\left(x_{2}\right)\right)\right)
$$

The nonlinear middle layer fusion model is as follows:

$$
f_{\eta_{m_{1,2}}}=\operatorname{Softmax}\left(w_{\eta}^{T} \operatorname{Relu}\left(w_{1}^{T} f_{m_{1}, l}+w_{2}^{T} f_{m_{2}, l}\right)\right)
$$

Theorem 1: The mutual information between the output of the fusion model $f_{\eta_{m_{1,2}}}$ and the true label $y$ for any of the three fusion methods is upper bounded by,

$$
\begin{aligned}
& \log \left(c_{1, t} e^{H\left(f_{1}\right)}+c_{2, t} e^{\left.\left(H\left(f_{2}, f_{1}\right)\right)\right)}+c_{3, t} e^{H\left(f_{2}\right)}\right)+ \\
& \log \left(c_{4, t} e^{\left(H\left(f_{1}, y\right)\right.}+c_{5, t} e^{\left.H\left(f_{2}, y\right)\right)}\right)+ \\
& H(y)+Z_{n}
\end{aligned}
$$

From the first line, we can clearly see the information gain is highly correlated to the log-linear of summation of each branch's entropy and the joint entropy between branches. The second line shows the information gain is also dependent on the cross-entropy of each branch models' predictions and the true labels. The third line would be a constant value given a fixed dataset.

Note that the proof of the non-linear middle fusion is based on the relaxation of the ReLU activation function with upper and lower bound [36]. More specifically, we replace the ReLU activations $h=\max (0, \hat{h})$ with their upper convex envelopes given a lower and upper bounds $l, u$, such that $h \geq 0, h \geq \hat{h}, u \hat{h}-u l \geq(u-l) h$. An example is shown in Fig. 2.

The theorem above leads to conjecture 1:

Conjecture 1: Given the fixed value of $H\left(f_{1}\right), H\left(f_{2}\right), H\left(f_{1}, y\right)$, and $H\left(f_{2}, y\right)$, the mutual information between the output of the fusion model $f_{m_{\eta}}$ and $y, I\left(f_{\eta_{m_{1,2}}}, y\right)$ is upper bound by a function of:

$$
I\left(f_{m_{\eta}}(X), y\right)=\text { const }+\log \left(\text { const }+ \text { const } * \exp \left(H\left(f_{1}, f_{2}\right)\right)\right)
$$

Conjecture 1 shows that, when the summation values of entropy and cross-entropy between predictions and labels for each branch candidate model are the same, the performance of the final fusion model is determined by the value of diversity distance between branches. Therefore, the fusion model will benefit from a considerable diversity between branch models. This validates the empirical observation in this research area.

\subsection{Multiple branches}

We further extend our theorem to $M(\geq 2)$ modalities for more complex situations. Now, the three fusion methods are rewritten as follows.

3.4.1 Late fusion method. Given model $f_{i}$ for each modality $i$, The late fusion method is constructed as,

$$
f_{m_{\eta}}(X)=\sum_{i=1}^{M} \beta_{i} f_{i}
$$

where $\sum_{i}^{M} \beta_{i}=1, \beta_{i} \in[0,1]$.

3.4.2 The middle fusion method. Similarly, the last latent layer of the branch model $f_{i}$ for modality $i$ is represented as $f_{m_{i}}$. The middle fusion method is constructed as,

$$
f_{m_{\eta}}(X)=\operatorname{Softmax}\left(\sum_{i=1}^{M} w_{i}^{T} f_{m_{i}, l}\right)
$$

3.4.3 The nonlinear middle fusion method. We also apply the ReLU non-linearity as the activation function. The nonlinear middle fusion method is constructed as,

$$
f_{m_{\eta}}(X)=\operatorname{Softmax}\left(w_{\eta}^{T} \operatorname{Relu}\left(\sum_{i=1}^{M} w_{i}^{T} f_{m_{i}, l}\right)\right)
$$

Theorem 2: For fusion method $\eta$, the mutual information between the fusion model $f_{\eta_{m_{1, \ldots, M}}}$ and the true label $y$ is upper bounded by,

$$
\begin{aligned}
& \log \left(\sum_{i=1}^{M} c_{i, t} e^{H\left(f_{i}\right)}+e^{c_{M+1, t} H\left(f_{i=1, \ldots, M}\right)}\right)+ \\
& \log \left(\sum_{i=1}^{M} c_{M+1+i, t} \exp \left(H\left(f_{i}, y\right)\right)\right)+ \\
& Z_{n}+H(y)
\end{aligned}
$$

Similar to Theorem 1, for cases with more than two modalities, the final fusion model's information gain is also a function of summation of all branch's entropy, the distance between branches (here, we calculate by total correlation between branches) of the final fusion model, and the original performance measure of the branch models, which is the cross-entropy of branch models' prediction and the true label.

Conjecture 2: Given the fixed value of $H\left(f_{i}\right), H\left(f_{i}, y\right)$, the mutual information between the output of the fusion model and $y$, $I\left(f_{m_{\eta}}, y\right)$ thus is bounded by:

$$
I\left(f_{m_{\eta}}(X), y\right)=\text { const }+\log \left(\exp \left(\text { const } * H\left(f_{1}, \ldots, f_{M}\right)\right)\right)
$$

Similar to conjecture 1, conjecture 2 validates the empirical observation that a multi-branch fusion model achieves a higher objective function when there is a high diversity among branches in a more generalized form. In the section.4, we will empirically show such log-linear correlation between fusion model's performance gain and the model distance in our experiment section. 


\subsection{Branch model selection framework}

Based on our theorem and our empirical observation of such correlation, in this section, we present our branch model selection framework that could efficiently select the branch combinations $\left\{\theta_{i, k} \mid 1 \leq k \leq K_{i}\right\}_{i=1, \ldots, M}$ for the final fusion model $f_{\eta_{m_{1, \ldots, M}}}$ to achieve potentially the highest objective function in an efficient manner. The framework has two basic modules: the diversity estimator to calculate the basic statistics of branch models, and the performance estimator to estimate the fusion model candidate's performance.

Instead of training all possible combinations $\prod_{i=1}^{M} K_{i}$ of fusion models, we use the performance estimation function from Theorem 1, 2 to aid the branch model selection. According to Theorem 1,2 , the final fusion model's mutual information is a function of each branch model's entropy, the diversity distance between these branch models, and the performance of each branch indicated by the cross-entropy. The estimation function thus can be obtained through the learning the variables in the function $c_{\cdot, t}$. To obtain the value of variables in the function, We first compute each branch model's entropy $H\left(f_{i}\right)$, cross entropy between model $f_{m_{i}, \theta_{i, k}}$ and $\mathrm{y}$ as $H\left(f_{m_{i}, \theta_{i, k}}, y\right)$, as well as the model diversity between models $H\left(f_{m_{1}, \theta_{1, k_{1}}}, \ldots, f_{m_{M}, \theta_{M, k_{M}}}\right)$ using the diversity evaluation module. Then, we train several randomly selected fusion models and calculate the performance gain $\operatorname{Acc}\left(f_{m_{\eta}}\right)-\max \left(\operatorname{Acc}\left(\left\{f_{i}\right\}_{i=1, \ldots, M}\right)\right)$. We use these data samples as training points, and perform the regression task to fit the factor $c_{\cdot, t}$ in Theorem 1 (if $M>2$, we fit Theorem 2 ) using the stochastic gradient descent method. The performance estimator module use this function to predict the potential performance.

After learning the factors $c_{\cdot, t}$ in the theorem, the performance estimator module infers the potential performance of all the fusion model candidates before training these candidates. We sort the ranking of these candidates according to the estimated potential performance. The top-K estimated-performance fusion models are selected as the pool of candidates for training. The value of $k$ depends on our computational resources.

Therefore, instead of training all $\prod_{i=1}^{M} K_{i}$ combination of fusion models, we only the train $k$ models, which save tons of training cost. The diagram of the model combination framework is shown in Figure. 3.

\section{EXPERIMENTS}

\subsection{Dataset}

To prove the effectiveness of our framework, we demonstrates our approach on three different tasks: e-Commerce advertisement (Ferramenta dataset), Kinetic-Sound prediction (Kinetic-sound dataset), sentimental analysis (CMU-YouTube, MOUD, MOSI datasets).

4.1.1 Ferramenta dataset. Ferramenta dataset $[15]^{1}$ consist of 52 classes (paintbrush, hinge, tape, safe, cart, etc.). There are 88010 images split in 66141 training images and 21869 test images. The text descriptions contain 22045 distinct words for the train set and 20083 for the test set. The dataset was collected from the price comparison website Trovaprezzi.

\footnotetext{
${ }^{1}$ https://github.com/artelab/Multi-modal-classification
}

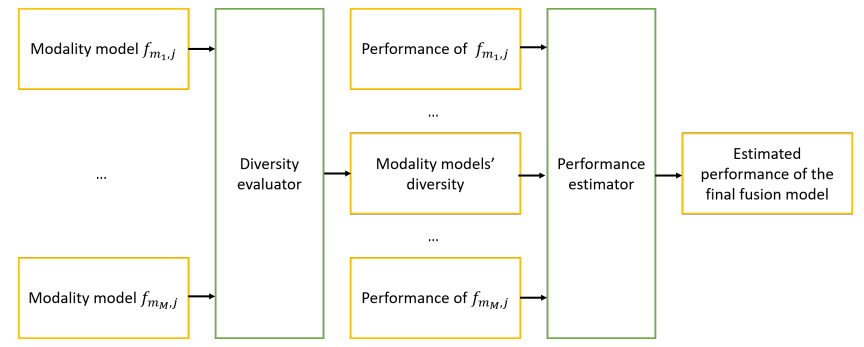

Figure 3: Workflow diagram of the framework. The framework takes in each branch's performance and distance measure between each branch's candidates. The performance estimator takes in the statistics of branch models and the diversity measure of these models and outputs the estimated performance. The top-K estimated performance model combinations are selected for final training.

4.1.2 Kinetic-sound dataset. Kinetics-sound dataset ${ }^{2}[4]$ is a subset of Kinetics [19] that contains 34 classes of audio-related events. There are 22,107 training videos and 1504 validation videos available when we download the videos. We train and evaluate multimodal (video and audio) event classification on Kinetics-sound. For the audio branch, we extract the same Mel filter bank features, which have 400 frames and $64 \mathrm{Mel}$-filter bins. For the visual branch, We use the $\mathrm{R}(2+1) \mathrm{D}$ network that computes convolutions across space and time for encoding the videos. The bottleneck features are input to the video branch.

4.1.3 CMU-YouTube Opinion Dataset . YouTube Opinion Dataset created by [24] is a Multimodal sentiment analysis datasets. It contains manually transcribed text, extracted audio, visual features, as well as the utterance. The dataset is further extended by [35] from 47 to $^{3} 70$ videos. $^{3}$

4.1.4 CMU-MOUD Dataset. The MOUD dataset[29] consists of product review videos in Spanish. Each video consists of multiple segments labeled to display positive, negative, or neutral sentiment.

4.1.5 CMU-MOSI Dataset. CMU Multimodal Opinion Sentiment Intensity (CMU-MOSI) is a dataset of opinion-level sentiment intensity in online videos. It contains 2199 opinion utterances with sentiment annotated between very negative to very positive.

\subsection{Model Architecture}

For the Ferramenta dataset, we design our model architecture based on the default textImgCNN model architecture in the Github repo 4 . For the image branch, we adjust the number of the conv layers, initializer, hidden channels, dropout rate. For the text branch, we change the number of fully connected layers, initializer, hidden units, dropout rate. For each branch, there are 54 pre-trained branch candidate models. The final fusion model is trained with Adam optimizer for 100 epochs with an early stop.

\footnotetext{
${ }^{2}$ https://github.com/Showmax/kinetics-downloader

${ }^{3}$ Datasets could be downloaded through: http://immortal.multicomp.cs.cmu.edu/raw datasets/processed data

${ }^{4}$ https://github.com/artelab/Multi-modal-classification
} 
For the other datasets, we construct the branch model architecture with the recurrent structure. The branch model recurrent cell type includes long short-term memory (LSTM), recurrent neural network cell (RNN), gated recurrent units (GRU), multi-headed attention as the backbone model. The number of recurrent layers ranges from 1 to 3 . The branch models are randomly initialized with a different initializer (Xavier, He). The choice for training hyperparameters includes hidden units $(60,80,100)$, dropout rate $(0.5$, $0.7,0.9)$, optimizer (Adam). All of these branch models are trained with 100 epochs with an early stop. The final fusion model is a combination of the selected branches. Thus, for each branch, there are $4 \times 3 \times 2 \times 3 \times 3=216$ pre-trained candidate models. The final fusion model is trained with Adam optimizer for 100 epochs with an early stop.

The hardware environment is configured with Intel Core i99920X , Nvidia-RTX8000, 128GB memory. The software environment is Ubuntu 18.04.5 LTS, CUDA 10.2, Pytorch.

\subsection{Correlation Between Model Distance and Accuracy Improvement}

In this subsection, we empirically show the correlation between model distance and accuracy improvement. We randomly select 120 final fusion models and train them on the CMU-Youtube, CMUMOUD, and CMU-MOSI dataset. We measure the exponent of the distance between the fusion model's branches $\exp \left(H\left(f_{1}, \ldots, f_{M}\right)\right)$, and calculate the difference between the fusion models' accuracy and the highest accuracy of the fusion models' branches as performance improvement $\operatorname{Acc}\left(f_{m_{\eta}}\right)-\max \left(\operatorname{Acc}\left(\left\{f_{i}\right\}_{i=1, \ldots, M}\right)\right)$. We fit the Spearman's correlation between these two variables using the 120 data samples for each dataset. The Spearman's correlation evaluates the non-linear relationships between the two variables. A perfect value of Spearman correlation of +1 or -1 indicates a perfect non-linear relation between the variables.

Fig. 4, 5, 6 shows the results of the correlation between the exponential joint entropy distance and the performance improvement for these three datasets. For all datasets, we obtained high Spearman correlation as high as 0.917 , which indicates that performance improvement is strongly non-linearly correlated with the exponent of the joint entropy distance. In other word, the larger the distance, the higher the performance improvement could be achieved. Such observation validates our theorems and conjectures, as well as the observations in the area.

\subsection{Framework's Performance}

In this subsection, we show the effectiveness of our framework. For each task, We select Top-120 fusion model candidates for final training according to their estimated performance predicted by our framework.

For a compare comparison, we fuse our candidates use the same fusion technique from the original works as baselines. The result is shown in Table 1 to Table 5. As shown in Table 1, the selected model could achieve an accuracy improvement as high as $2.6 \%$ compared to the direct fusion model using the late/middle/nonlinear fusion method we analyzed in our paper. In Table 2, our selected model could also achieve an accuracy improvement of $1.7 \%$ for other specially design fusion method.

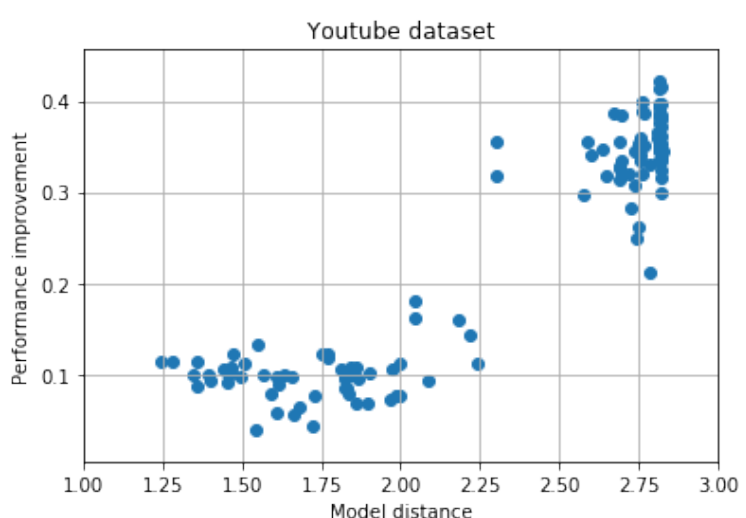

Figure 4: CMU-Youtube dataset: Performance improvement vs joint entropy distance gives a Spearman correlation factor of 0.832 . p-value $=2.5 \mathrm{e}-28$.

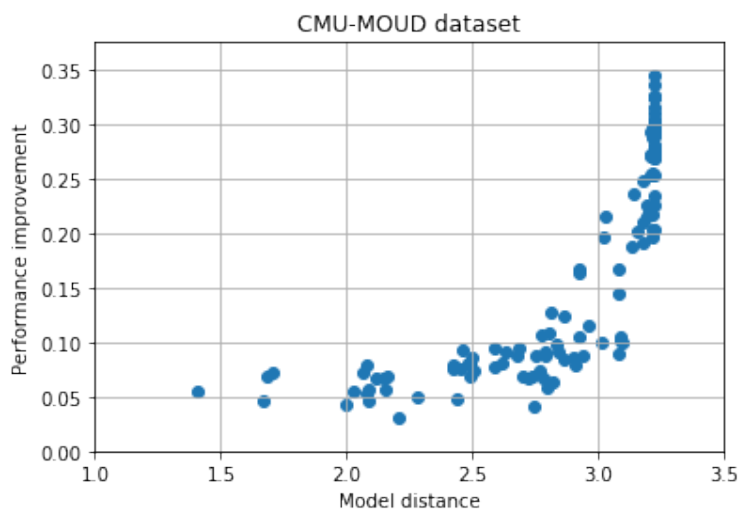

Figure 5: CMU-MOUD dataset: Spearman correlation factor of $0.917 . p$ value $=3.6 \mathrm{e}-45$.

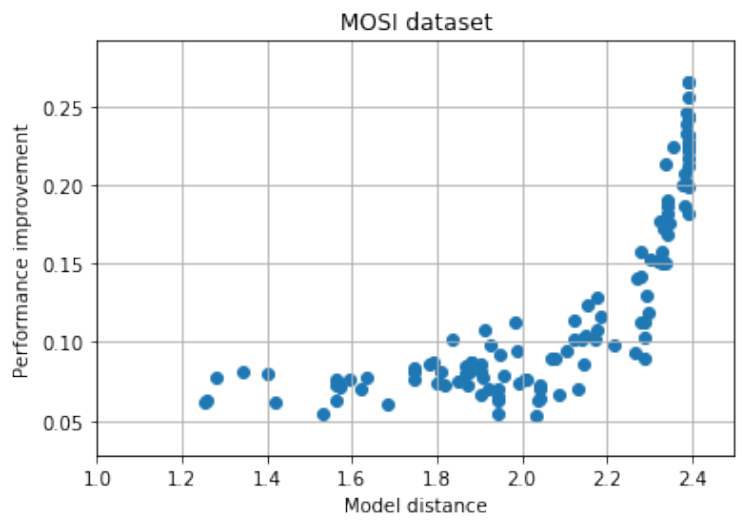

Figure 6: CMU-MOSI dataset: Spearman correlation factor of 0.887 and $p$-value $=1.7 e-39$.

Through these results, we could easily find the empirical validation of the effectiveness of the framework: the fusion model's 


\begin{tabular}{lrrrr}
\hline $\begin{array}{c}\text { Fusion } \\
\text { Method }\end{array}$ & $\begin{array}{c}\text { Selected } \\
\text { fusion's Acc }\end{array}$ & $\begin{array}{c}\text { Selected } \\
\text { fusion's F1 }\end{array}$ & $\begin{array}{c}\text { Direct fusion } \\
\text { Acc. }\end{array}$ & $\begin{array}{c}\text { Direct fusion } \\
\text { F1. }\end{array}$ \\
\hline DS & 0.944 & 0.944 & 0.937 & 0.937 \\
LOP & $\mathbf{0 . 9 5 9}$ & $\mathbf{0 . 9 5 9}$ & 0.944 & 0.944 \\
S-L & 0.912 & 0.912 & 0.895 & 0.895 \\
S-MLP & 0.958 & 0.958 & 0.932 & 0.932 \\
\hline
\end{tabular}

Table 1: Performance of our best performing fusion models shown in Ferramenta dataset. The previous paper uses four fusion methods [14]: distribution summation (DS), which is similar to our late fusion; logarithmic Opinion Pool (LOP); Logistic regression(S-L), which is similar to our middle fusion; and Multi-layer Perceptron(S-MLP), similar to nonlinear middle fusion. Direct fusion refers to the higher performance of the fusion model that directly fuses the bestperforming branch models or the claim by the original paper.

performance depends both high diversity together with high performance of branch models. The fusion models whose branch models are selected by the framework achieve superior performance than the previous fusion model's result that directly fuse the bestperforming branch models. We could see the clear benefit of our proposed framework, which could help to efficiently find the combination branch model candidates to fuse and achieve high-accuracy with improvements on all five datasets.

As noticed, besides the late/middle/non-linear fusion methods we analyzed([14] called them as distribution summation, logistic regression, multi-layer perceptron), our proposed branch model selection framework also works well with other fusion methods including fusion network[16], tensor fusion networks[41] and etc.

\begin{tabular}{lrrrr}
\hline $\begin{array}{c}\text { Fusion } \\
\text { Method }\end{array}$ & $\begin{array}{c}\text { Selected } \\
\text { fusion's Acc }\end{array}$ & $\begin{array}{c}\text { Selected } \\
\text { fusion's mAP }\end{array}$ & $\begin{array}{c}\text { Direct fusion } \\
\text { Acc. }\end{array}$ & $\begin{array}{c}\text { Direct fusion } \\
\text { mAP. }\end{array}$ \\
\hline FN & 0.816 & 0.827 & 0.810 & 0.810 \\
AVSlowFast & $\mathbf{0 . 8 6 7}$ & $\mathbf{0 . 8 6 8}$ & 0.850 & 0.849 \\
S-MLP & 0.854 & 0.855 & 0.834 & 0.834 \\
\hline
\end{tabular}

Table 2: Performance of our best performing fusion models shown in the kinetics-sound dataset. We apply our framework to Fusion Network (FN) [16], Audio-Video Slow Fast Pathway (AVSlowFast) [38] and S-MLP fusion methods.

\subsection{Discussion}

In this subsection, we show the estimated performance of fusion models using our framework. The result is based on the CMU-MOSI dataset. Table 6 shows the performance of each branch model, the estimated performance, and the final accuracy. As we can see from Table 6, the highest branch model's performance is $47.42 \%$ for covarep, $79.98 \%$ for text, and $51.50 \%$ for facet, while the performance of the combination of these models are inferior to other candidates. The combination of the highest-performing branch candidate models does not necessarily guarantee the highest final fusion model's

\begin{tabular}{lrrrr}
\hline $\begin{array}{c}\text { Fusion } \\
\text { Method }\end{array}$ & $\begin{array}{c}\text { Selected } \\
\text { fusion's Acc }\end{array}$ & $\begin{array}{c}\text { Selected } \\
\text { fusion's F1 }\end{array}$ & $\begin{array}{c}\text { Direct fusion } \\
\text { Acc. }\end{array}$ & $\begin{array}{c}\text { Direct fusion } \\
\text { F1. }\end{array}$ \\
\hline TFN & - & - & 0.475 & 0.410 \\
MARN & - & - & 0.542 & 0.529 \\
S-MLP & $\mathbf{0 . 8 5 6}$ & $\mathbf{0 . 8 5 6}$ & 0.823 & 0.822 \\
\hline
\end{tabular}

Table 3: Performance of our best performing fusion models shown in CMU-Youtube. We apply our framework to SMLP fusion method, and compare to Tensor Fusion Network (TFN) [41], Multi-attention Recurrent Network (MARN) [43]. The performance of our model using non-linear fusion is higher than these fusion method. One of the reason might because that we trained our model on the preprocessed features provided by the organizer.

\begin{tabular}{lrrrr}
\hline $\begin{array}{c}\text { Fusion } \\
\text { Method }\end{array}$ & $\begin{array}{c}\text { Selected } \\
\text { fusion's Acc }\end{array}$ & $\begin{array}{c}\text { Selected } \\
\text { fusion's F1 }\end{array}$ & $\begin{array}{c}\text { Direct fusion } \\
\text { Acc. }\end{array}$ & $\begin{array}{c}\text { Direct fusion } \\
\text { F1. }\end{array}$ \\
\hline TFN & - & - & 0.632 & 0.617 \\
MARN & - & - & 0.811 & 0.812 \\
S-MLP & $\mathbf{0 . 9 0 6}$ & $\mathbf{0 . 9 0 6}$ & 0.888 & 0.889 \\
\hline
\end{tabular}

Table 4: Performance of our best performing fusion models shown in CMU-MOUD. We apply our framework to S-MLP fusion method, and compare to TFN and MARN methods.

\begin{tabular}{lrrrr}
\hline $\begin{array}{c}\text { Fusion } \\
\text { Method }\end{array}$ & $\begin{array}{c}\text { Selected } \\
\text { fusion's Acc }\end{array}$ & $\begin{array}{c}\text { Selected } \\
\text { fusion's F1 }\end{array}$ & $\begin{array}{c}\text { Direct fusion } \\
\text { Acc. }\end{array}$ & $\begin{array}{c}\text { Direct fusion } \\
\text { F1. }\end{array}$ \\
\hline TFN & - & - & 0.745 & 0.745 \\
MARN & - & - & 0.770 & 0.770 \\
S-MLP & $\mathbf{0 . 8 5 6}$ & $\mathbf{0 . 8 5 7}$ & 0.849 & 0.850 \\
Human & - & - & 0.875 & 0.875 \\
\hline
\end{tabular}

Table 5: Performance of our best performing fusion models shown in CMU-MOSI. The model generated by our framework is much higher than the baseline, and close to human performance.

accuracy. ${ }^{5}$ Also, we find that the estimated performance is relatively close to the final training performance with a deviation of $\pm 2 \%$. This further shows the effectiveness of our framework.

\begin{tabular}{lrrrrr}
\hline Rank & Covarep & Text & Facet & Fusion model & Est. Perf. \\
\hline 1 & 0.3988 & 0.7783 & 0.5000 & $\mathbf{0 . 8 5 6 7}$ & 0.8427 \\
2 & 0.4338 & 0.7998 & 0.5000 & 0.8492 & 0.8532 \\
3 & 0.4113 & 0.7959 & 0.4924 & 0.8464 & 0.8395 \\
12 & $\mathbf{0 . 4 7 4 2}$ & $\mathbf{0 . 7 9 9 8}$ & $\mathbf{0 . 5 1 5 0}$ & 0.8222 & 0.8314 \\
\hline
\end{tabular}

Table 6: Top-3 performance fusion models' accuracy, their branch model's (Covarep, Text, facet) accuracy in CMUMOSI dataset, and the estimate performance, together with the result of the fusion model that fuses the bestperforming branch models.

\footnotetext{
${ }^{5}$ The same evidence is observed in other datasets
} 


\section{CONCLUSION}

In this paper, we propose the diversity analysis for multi-branch fusion models in the multi-modal machine learning area toward a higher performance on multi-modal multi-class classification problems. We introduce a way to measure the diversity between branch models. We theoretically prove that the final fusion model performance is a function of the distance between branch models and the branch model's performance indicated by the cross-entropy. The final model's performance gain has a log-linear correlation with the distance of these branch models. Based on our theorem, we propose a branch model selection framework that can efficiently identify branch model combinations to fuse and achieve higher accuracy compare to simply fusing the best-performing branch models. We validate our theorem by showing a high Spearman correlation between performance gain and the model distance, and the effectiveness of the framework by achieving competitive performance across five datasets on three different tasks.

\section{REFERENCES}

[1] Amir H Abdi, Purang Abolmaesumi, and Sidney Fels. 2019. A Preliminary Study of Disentanglement With Insights on the Inadequacy of Metrics. arXiv preprint arXiv:1911.11791 (2019)

[2] Galen Andrew, Raman Arora, Jeff Bilmes, and Karen Livescu. 2013. Deep canonical correlation analysis. In International conference on machine learning. PMLR, 12471255.

[3] Stanislaw Antol, Aishwarya Agrawal, Jiasen Lu, Margaret Mitchell, Dhruv Batra, C Lawrence Zitnick, and Devi Parikh. 2015. Vqa: Visual question answering. In Proceedings of the IEEE international conference on computer vision. 2425-2433.

[4] Relja Arandjelovic and Andrew Zisserman. 2017. Look, listen and learn. In Proceedings of the IEEE International Conference on Computer Vision. 609-617.

[5] John Arevalo, Thamar Solorio, Manuel Montes-y Gómez, and Fabio A González. 2017. Gated multimodal units for information fusion. arXiv preprint arXiv:1702.01992 (2017)

[6] Pradeep K Atrey, M Anwar Hossain, Abdulmotaleb El Saddik, and Mohan S Kankanhalli. 2010. Multimodal fusion for multimedia analysis: a survey. Multimedia systems 16, 6 (2010), 345-379.

[7] Pierre Baldi, Søren Brunak, Yves Chauvin, Claus AF Andersen, and Henrik Nielsen. 2000. Assessing the accuracy of prediction algorithms for classification: an overview. Bioinformatics 16, 5 (2000), 412-424.

[8] Tadas Baltrušaitis, Chaitanya Ahuja, and Louis-Philippe Morency. 2018. Multimodal machine learning: A survey and taxonomy. IEEE transactions on pattern analysis and machine intelligence 41, 2 (2018), 423-443.

[9] Mohamed Ishmael Belghazi, Aristide Baratin, Sai Rajeswar, Sherjil Ozair, Yoshua Bengio, Aaron Courville, and R Devon Hjelm. 2018. Mine: mutual information neural estimation. arXiv preprint arXiv:1801.04062 (2018).

[10] Yoshua Bengio, Aaron Courville, and Pascal Vincent. 2013. Representation learning: A review and new perspectives. IEEE transactions on pattern analysis and machine intelligence 35,8 (2013), 1798-1828.

[11] Ricky TQ Chen, Xuechen Li, Roger B Grosse, and David K Duvenaud. 2018 Isolating sources of disentanglement in variational autoencoders. In Advances in Neural Information Processing Systems. 2610-2620.

[12] Desmond Elliott and Akos Kádár. 2017. Imagination improves multimodal translation. arXiv preprint arXiv:1705.04350 (2017).

[13] Fangxiang Feng, Xiaojie Wang, and Ruifan Li. 2014. Cross-modal retrieval with correspondence autoencoder. In Proceedings of the 22nd ACM international conference on Multimedia. 7-16.

[14] Ignazio Gallo, Alessandro Calefati, and Shah Nawaz. 2017. Multimodal classification fusion in real-world scenarios. In 2017 14th IAPR International Conference on Document Analysis and Recognition (ICDAR), Vol. 5. IEEE, 36-41.

[15] I. Gallo, A. Calefati, and S. Nawaz. 2017. Multimodal Classification Fusion in RealWorld Scenarios. In 2017 14th IAPR International Conference on Document Analysis and Recognition (ICDAR), Vol. 05. 36-41. https://doi.org/10.1109/ICDAR.2017.326

[16] Ruohan Gao, Tae-Hyun Oh, Kristen Grauman, and Lorenzo Torresani. 2020 Listen to look: Action recognition by previewing audio. In Proceedings of the IEEE/CVF Conference on Computer Vision and Pattern Recognition. 10457-10467.

[17] Zhiqiang Gong, Ping Zhong, and Weidong Hu. 2019. Diversity in machine learning. IEEE Access 7 (2019), 64323-64350.

[18] Zhenhua Huang, Xin Xu, Juan Ni, Honghao Zhu, and Cheng Wang. 2019. Multimodal representation learning for recommendation in Internet of Things. IEEE Internet of Things fournal 6, 6 (2019), 10675-10685.
[19] Will Kay, Joao Carreira, Karen Simonyan, Brian Zhang, Chloe Hillier, Sudheendra Vijayanarasimhan, Fabio Viola, Tim Green, Trevor Back, Paul Natsev, et al. 2017. The kinetics human action video dataset. arXiv preprint arXiv:1705.06950 (2017).

[20] Douwe Kiela, Edouard Grave, Armand Joulin, and Tomas Mikolov. 2018. Efficient large-scale multi-modal classification. In Proceedings of the AAAI Conference on Artificial Intelligence, Vol. 32.

[21] Yann LeCun, Yoshua Bengio, and Geoffrey Hinton. 2015. Deep learning. nature 521, 7553 (2015), 436-444.

[22] Philip A Legg, Paul L Rosin, David Marshall, and James E Morgan. 2013. Improving accuracy and efficiency of mutual information for multi-modal retinal image registration using adaptive probability density estimation. Computerized Medical Imaging and Graphics 37, 7-8 (2013), 597-606.

[23] Zhun Liu, Ying Shen, Varun Bharadhwaj Lakshminarasimhan, Paul Pu Liang, Amir Zadeh, and Louis-Philippe Morency. 2018. Efficient low-rank multimodal fusion with modality-specific factors. arXiv preprint arXiv:1806.00064 (2018).

[24] Louis-Philippe Morency, Rada Mihalcea, and Payal Doshi. 2011. Towards multimodal sentiment analysis: Harvesting opinions from the web. In Proceedings of the 13th international conference on multimodal interfaces. 169-176.

[25] Vinod Nair and Geoffrey E Hinton. 2010. Rectified linear units improve restricted boltzmann machines. In ICML.

[26] Jiquan Ngiam, Aditya Khosla, Mingyu Kim, Juhan Nam, Honglak Lee, and Andrew Y Ng. 2011. Multimodal deep learning. In ICML.

[27] Soujanya Poria, Erik Cambria, Rajiv Bajpai, and Amir Hussain. 2017. A review of affective computing: From unimodal analysis to multimodal fusion. Information Fusion 37 (2017), 98-125.

[28] Seyed Mahdi Roostaiyan, Ehsan Imani, and Mahdieh Soleymani Baghshah. 2017. Multi-modal deep distance metric learning. Intelligent Data Analysis 21, 6 (2017), 1351-1369.

[29] Verónica Pérez Rosas, Rada Mihalcea, and Louis-Philippe Morency. 2013. Multimodal sentiment analysis of spanish online videos. IEEE Intelligent Systems 28, 3 (2013), 38-45.

[30] Kihyuk Sohn, Wenling Shang, and Honglak Lee. 2014. Improved multimodal deep learning with variation of information. Advances in neural information processing systems 27 (2014), 2141-2149.

[31] Nitish Srivastava, Ruslan Salakhutdinov, et al. 2012. Multimodal Learning with Deep Boltzmann Machines.. In NIPS, Vol. 1. Citeseer, 2.

[32] Bruce Thompson. 2005. Canonical correlation analysis. Encyclopedia of statistics in behavioral science (2005).

[33] Aleksei Tiulpin, Stefan Klein, Sita MA Bierma-Zeinstra, Jérôme Thevenot, Esa Rahtu, Joyce van Meurs, Edwin HG Oei, and Simo Saarakkala. 2019. Multimodal machine learning-based knee osteoarthritis progression prediction from plain radiographs and clinical data. Scientific reports 9, 1 (2019), 1-11.

[34] Viivi Uurtio, Sahely Bhadra, and Juho Rousu. 2019. Large-scale sparse kernel canonical correlation analysis. In International Conference on Machine Learning. PMLR, 6383-6391.

[35] Martin Wöllmer, Moritz Kaiser, Florian Eyben, Björn Schuller, and Gerhard Rigoll. 2013. LSTM-Modeling of continuous emotions in an audiovisual affect recognition framework. Image and Vision Computing 31, 2 (2013), 153-163.

[36] Eric Wong and Zico Kolter. 2018. Provable defenses against adversarial examples via the convex outer adversarial polytope. In International Conference on Machine Learning. PMLR, 5286-5295.

[37] Yi Wu, Edward Y Chang, Kevin Chen-Chuan Chang, and John R Smith. 2004. Optimal multimodal fusion for multimedia data analysis. In Proceedings of the 12th annual ACM international conference on Multimedia. 572-579.

[38] Fanyi Xiao, Yong Jae Lee, Kristen Grauman, Jitendra Malik, and Christoph Feichtenhofer. 2020. Audiovisual SlowFast Networks for Video Recognition. arXiv preprint arXiv:2001.08740 (2020)

[39] Pengtao Xie and Eric P Xing. 2013. Multi-modal distance metric learning. In Proceedings of the Twenty-Third international joint conference on Artificial Intelligence. AAAI Press, 1806-1812.

[40] Ting Yao, Yingwei Pan, Yehao Li, Zhaofan Qiu, and Tao Mei. 2017. Boosting image captioning with attributes. In Proceedings of the IEEE International Conference on Computer Vision. 4894-4902.

[41] Amir Zadeh, Minghai Chen, Soujanya Poria, Erik Cambria, and Louis-Philippe Morency. 2017. Tensor fusion network for multimodal sentiment analysis. arXiv preprint arXiv:1707.07250 (2017)

[42] Amir Zadeh, Paul Pu Liang, Navonil Mazumder, Soujanya Poria, Erik Cambria, and Louis-Philippe Morency. 2018. Memory fusion network for multi-view sequential learning. arXiv preprint arXiv:1802.00927 (2018)

[43] Amir Zadeh, Paul Pu Liang, Soujanya Poria, Prateek Vij, Erik Cambria, and Louis-Philippe Morency. 2018. Multi-attention recurrent network for human communication comprehension. In Proceedings of the AAAI Conference on Artificial Intelligence, Vol. 32

[44] Jun-Yan Zhu, Richard Zhang, Deepak Pathak, Trevor Darrell, Alexei A Efros, Oliver Wang, and Eli Shechtman. 2017. Toward multimodal image-to-image translation. arXiv preprint arXiv:1711.11586 (2017). 